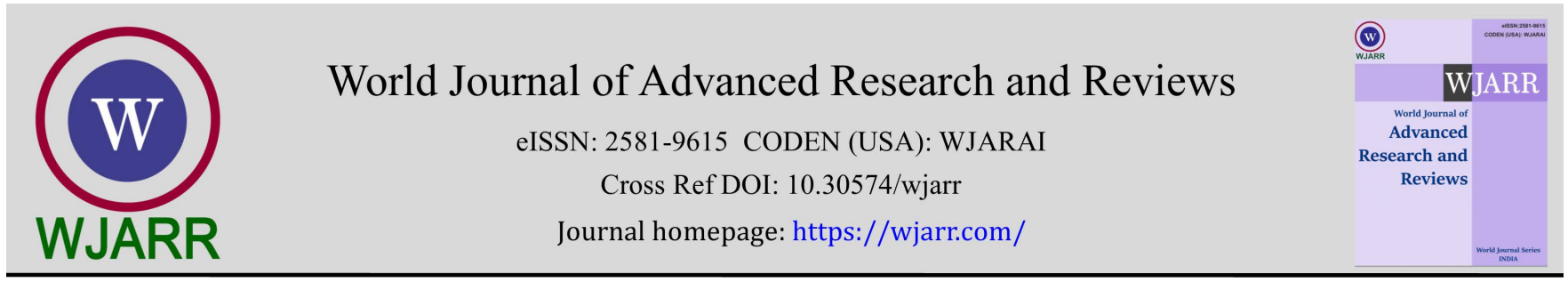

(SHORT COMMUNiCATION)

\title{
Oppositional defiant disorder and Chinese music therapy: The 4S-Model
}

\author{
Wolfgang Mastnak* \\ Beijing Normal University BNU. \\ World Journal of Advanced Research and Reviews, 2022, 13(01), 481-486
}

Publication history: Received on 19 November 2021; revised on 10 January 2022; accepted on 12 January 2022

Article DOI: https://doi.org/10.30574/wjarr.2022.13.1.0763

\begin{abstract}
Oppositional defiant disorder (ODD) is characterised by recurrent patterns of developmentally inappropriate, negativistic, defiant, hostile, annoying and disobedient behaviour, mainly toward people in authority, and it may increase the risk of delinquency. Notwithstanding the wide variation of relevant statistical data, ODD can be considered a serious global issue - and it is one of the most prevalent psychiatric diseases among Chinese children and adolescents. Mental health is a key objective of the Chinese ministry of education, and a declaration issued in 2021 calls for enhanced mental health education in primary and secondary schools. In consonance with these objectives, the Research Centre for Arts Therapies of Beijing Normal University is developing arts-based models to strengthen mental health, improve prevention of psycho-affective disorders and alleviate (subclinical) symptoms in the younger generation, ODD included. Regarding the pathological features of ODD, the present article suggests to notably regard the related dynamic complex of intrinsic factors, socio-cultural interdependencies and challenging personality traits. Moreover, it introduces the 4Smodel of Chinese music therapy for ODD consisting of (i) self-discovery, e.g. through aesthetic mirror techniques, (ii) self-control, e.g. involving creative martial arts, (iii) self-actualisation, e.g. based on sound-scene improvisation and (iv) self-adjustment, e.g. by means of music-induced trance and imagination techniques. Further research to optimise cultural sensitivity, e.g. concerning the ethnic minorities living in China, and to evaluate effect sizes are needed, alongside implementation into regular curricula and nationwide in-service-trainings for teachers.
\end{abstract}

Keywords: Chinese public health; Cross-cultural psychiatry; Cultural sensitivity; Educational music therapy; Mindfulness; Self-regulation techniques.

\section{Introduction}

To enhance mental health in the younger generation, the Chinese ministry of education issued a resolution [1], in Chinese 中小学要将心理健康教育课纳入校本课程, which calls for enhanced mental health education within regular curricula and classes in primary and secondary schools. This also involves preventive medical issues, interdisciplinary psycho-educational support of children and adolescents with subclinical symptoms, as well as pupils with manifest medical conditions in inclusive education. Beijing Normal University (BNU) is the leading educational university in China and its Research Centre for Arts Therapies - in Chinese 北京师范大学艺术治疗研究中心 - is focusing on a broad spectrum of culturally sensitive arts therapies, mental health issues in the younger Chinese generation included.

Oppositional defiant disorder is considered to be one of the most frequent psychiatric issues in Chinese children and adolescents. For instance, the twelve-month prevalence rate of ODD in central Hunan is estimated with 2.98\% [2]. Discussing possible sociocultural differences in ODD-distributions, a comparative analysis [3] highlights that contrary to what the authors expected, geographic location of the studies, which is usually considered a broad index of different cultures and contexts, was not associated with significant variability in prevalence estimates of conduct disorders (CD) or ODD. However, a study from Beijing Normal University [4] examined the relationship of oppositional defiant disorder

\footnotetext{
${ }^{*}$ Corresponding author: Wolfgang Mastnak

Beijing Normal University BNU .

Copyright (C) 2022 Author(s) retain the copyright of this article. This article is published under the terms of the Creative Commons Attribution Liscense 4.0.
} 
symptoms and factors at whole family, dyadic and individual levels in Chinese children and highlighted that family cohesion/adaptability was indirectly associated with ODD symptoms via parent-child relationship and child emotion regulation.

History of medicine sheds light on typical issues connected with the discovery and identification of psychiatric diseases, particularly the recognition of pathologically abnormal behaviour which differs from (just) unusual personality traits. The evolution of the diagnosis of ODD is interconnected with clinical studies on delinquent boys and factor analyses of their personalities [5, 6], and Pardini et al. [7] pointed out that the foundation for contemporary conceptualisations of ODD and CD came from the inclusion of childhood and adolescent disorders associated with delinquency in the $2^{\text {nd }}$ edition of the Diagnostic and Statistical Manual of Mental Disorders in 1968: 'Three theoretically distinct diagnoses were described as arising from divergent environmental factors: runaway reaction, unsocialized aggressive reaction, and group delinquent reaction'.

Due to significant intersections of ODD-symptoms and forensic psychiatry, the diagnosis has considerable discriminating power, hence the call for clear medical identification of pathological features [8]: ODD is often comorbid with attention-deficit/hyperactivity disorder, conduct disorder, and mood disorders, including anxiety and depression. Moreover, adults and adolescents with a history of ODD have a greater than $90 \%$ chance of being diagnosed with another mental illness in their lifetime. They are at high risk of developing social and emotional problems, including suicide and substance use disorders. Early intervention seeks to prevent the development of conduct disorder, substance abuse and delinquency that can cause lifelong social, occupational and academic impairments - and these are also key objectives of our actual music therapeutic model.

Conduct disorders are usually considered moderately heritable psychiatric disorders of childhood and adolescence [9] - and genetic influences on the development of oppositional defiant disorders are discussed alongside related risks for later psychopathology [10]. Nonetheless, the exact causes of ODD are still unknown and a combination of genetic, neurological, e.g. brain injury [11], and environmental factors is generally assumed.

Treatment of ODD is usually complex and may include psychotherapeutic interventions such as the Parent-Child Interaction Therapy, the Triple P-Positive Parenting Program, the Parent Management Training Oregon Model, Problem Solving Skills Training or Anger Control Training, alongside pharmacological treatment such as atypical antipsychotics - although medication is usually not considered a first line intervention for ODD, but rather a secondary treatment option [12]. The present article presents a completely different therapeutic model, which may be used as add-ontherapy, an educational support or even primary therapy.

\section{Material and methods}

Broadly speaking, medicine and clinical practice are dealing with three key issues: therapeutic media, underlying mechanisms, and estimation of effect sizes. Owing to advanced and highly specialised disciplines, medicine has lost important core areas: pharmacological research is in charge of the development of drugs, medical electronics produce devices such as intelligent pace-makers, and biochemistry or cognitive neurosciences discover underlying mechanisms. Together with the call for evidence based medicine, medical research has somehow lost its wholeness and balanced complexity. The importance of standardised designs such as randomised controlled trials and the statistical estimation of effect sizes are widely overestimated, and apparently blind belief in the hierarchical pyramid of evidence based medicine is likely to ignore or exclude meta-theoretical considerations, hence the occurrence of systemic errors that are masked by terms such as 'significance' or 'robustness'.

In this context, scientific epistemology raises the correspondence theoretical question how adequately abstract constructs, e.g. models based on numerical results from psychiatric inventories, represent the source objects referred to, alongside the issue whether or to what extent inferential statistics can be used to estimate non-mathematical entities, hence the new parameters of coherence size and confidence range [13].

The enormous shift towards evidence based medicine and the dominance of effect sizes in medical research have not only resulted in a biased orientation of medical journals, but probably also brought about a decline in inventive medicine, alongside a lack of new non-technical and non-pharmacological treatment. And while methods to estimate and substantiate effect sizes such as meta-analyses of RCTs have become a broadly accepted and well-known standard, scientific means to develop psychiatric interventions that are precisely tailored to new challenges, for instance, seem to fade into oblivion. 
Systemic meta-syntheses [14] are designed to construct new theories and to generate 'powered hypotheses', a process which can be considered complementary to the estimation of effect sizes as well as hypothesis-testing. In short: the various concepts of verification, power and truth matter. Akin to the concept of degrees of corroboration, the notion 'powered hypothesis' is based on epistemological strength: (i) similar to meta-analyses systemic meta-syntheses carefully select their components; different from meta-analyses, however, they are chosen because of their epistemological value and trans-disciplinary 'goodness of fit'; (ii) while the weight of a meta-analysis stems from the amount and methodological quality of the studies referred to, meta-syntheses depend on the quality of the linkage and how this blends with the inner logic of the entire framework; (iii) in line with the Gestalt-theoretical argument that the whole is greater than the sum of its parts, the theory of systemic meta-syntheses argues that the outcome of the whole study in not a simple and direct consequence of their parts, but substantially involves the mode of connecting its components and the epistemological rationale behind the synthesis, hence the term 'powered hypothesis', which also applies to the present study.

\section{Results and discussion}

A study on ODD in educational contexts [15] qualifies the common standpoint that symptoms of oppositional defiant disorder (ODD) reflect both a general diagnostic construct and two distinct symptom dimensions, namely irritability and defiant behaviour, and suggests a broad spectrum of pathological patterns including 'proactive and reactive aggression, relational and physical aggression and victimization, withdrawn/depressed symptoms, peer rejection, and academic performance.'

\subsection{A dynamic model of ODD}

In accordance with these findings, the present study also involves interdisciplinary integrative medical perspectives and suggests an open dynamic model of oppositional defiant disorder that is based on three cornerstones: sociocultural dynamics, multi-pathological dynamics and intrapersonal dynamics.

The diagnosis of ODD essentially involves interpretations of adverse behaviour such as being defiant and hostile toward peers, parents, teachers and authority figures in general. These, however, depend on sociocultural norms, conventions and inherent ethics and make the diagnosis of ODD a dependent variable. Moreover, sociocultural conditions may cause ODD-typical responses, particularly if individuals feel oppressed, over-controlled and patronised, so that the children's behaviour has to be understood as a sort of self-defence or the cry of a suffocating soul.

Oppositional defiant disorder is often comorbid with attention deficit hyperactivity disorder, conduct disorder, mood disturbances such as depression, anxiety disorders, as well as symptoms of autism or schizophrenia spectrum disorders $[8,16]$. Such findings are not consistent with the assumption of a clear-cut diagnosis of ODD and bring about philosophical medical questions about diseases as entities or phenomena. Not least for methodological and therapeutic reasons we suggest to (also) consider ODD as the expression of various patterns of genetic/epigenetic, neurobiological and psychological aberrations and dysfunctions [17].

In light of comparative considerations and interdisciplinary meta-syntheses we also encourage to discuss ODD as a socioculturally incompatible personality trait or the behavioural expression of an individually unique self, hence the ethical question about pathological stigmatisation. The inherent conflict between standardised psychiatric diagnostics and mindful humanistic standpoints, such as the paradigms of inclusive education, calls for interdisciplinary systemic approaches, both to support the social equilibrium and to avoid the destruction of 'peculiar children'.

\subsection{The Four-S-Model}

Considering oppositional defiant disorder a dynamic and systemic issue, we suggest a resource oriented treatment model that is based on mindful empowerment and arts-therapeutic approaches. It is designed for both educational and clinical use and shall help to develop the strengths of ODD-affected individuals and reduce their risk of social exclusion and stigmatisation. The four ' $\mathrm{S}$ ' stand for self-discovery, self-control, self-actualisation and self-adjustment.

\subsubsection{Self-discovery}

Although children and adolescents with ODD are frequently faced with derogatory labelling, these adverse stimuli do not reduce their irritating behaviour, but seem to work, paradoxically, as a sort of reinforcement, which tends to even increase the gap between the self and its social environment. Moreover, this perpetual stigmatisation may impact on the individual's self-image, and educational as well as therapeutic support to discover positive features of one's personality gains importance. 


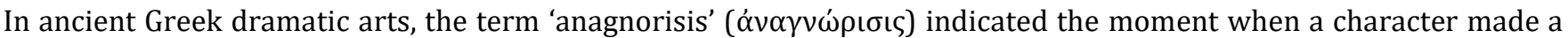
critical psychological or psycho-social discovery, recognition of one's true character included. Modern clinical approaches such as psychodramatic mirror techniques also refer to this principle, which likewise applies to today's cinema- and film-therapy $[18,19]$. The Four-S-Model encourages realistic and mindful self-discovery through such nondirective methods.

By way of illustration, from the perspective of today's psychopathology the protagonist, a naughty child, in Maurice Ravel's opera 'L'enfant et les sortilèges: Fantaisie lyrique en deux parties' [The Child and the Spells: A Lyric Fantasy in Two Parts] could be diagnosed with ODD [20]. Opposing his mother's rebuke, the child throws a tantrum, destroys his room and hurts animals nearby. The 'illusionary discovery' begins: the destroyed objects come to life and want to punish him for his misdeeds. During a fight among animals the child eventually discovers his sense of compassion and rescues a hurt squirrel, the moment where his self-image, behaviour and social integration change.

\subsubsection{Self-control}

Martial arts are also discussed as a way to achieve self-control in individuals with disruptive behaviour disorders - and transforming aggression and oppositional behaviour through combat sports is considered a viable means to enhance crime prevention [21]. To increase self-control and cultivate self-discipline, the present article suggests activities that (i) facilitate one's experience of self-identity, which also improves adherence and sustainability [22], (ii) require regular training alongside motivating feedback-control, (iii) involve physical exertion and strengthen cardiorespiratory fitness, and (iv) allow artistic transformation of inner impulses and affective processes.

Regarding Chinese cultural backgrounds and traditions, appropriate activities may include Chinese percussion such as the Daluo 大锣, a large gong, the Yunluo 云锣, an instrument with many small gongs, the Bianzhong 编钟, 16 to 65 bronze bells hung on a rack, alongside a multitude of different drums, as well as unique instruments such as the fish shaped woodblock Muyu 木鱼 - or creative Qigong, which also involves vocal expression.

\subsubsection{Self-actualisation}

We assume that oppositional defiant disorder may be caused by an experienced imbalance between external behaviour determination and a strong incentive to self-actualisation, which leads to escalating conflicts and further repression, which repeatedly trigger typical reactions and result in an individually detrimental vicious circle.

In the sense of Maslow's hierarchy of needs, self-actualisation means the complete realisation of one's potential, as well as the individual's ideal appreciation of life. Although widely according with Maslow's concept of self-actualisation, this article does not agree with Maslow's assumption that the internal drive to self-actualise would seldom emerge until more basic needs are met. In our opinion, self-actualisation is deeply interconnected with self-identity and particularly in individuals with an ODD diagnosis an explosive driving force.

Although self-actualisation is highly individual, there are frames that facilitate both the vision and the experience of selfactualisation - one of them is sound scene improvisation. Sound scene improvisation is a core model of Polyaesthetic Education [23], an integrative music educational concept that also integrates drama, dance, poetry and the fine arts. Polyaesthetic Education goes back to the seminal work of Wolfgang Roscher, who emphasised the developmental and educational value of artistic improvisation, and music-dramatic improvisation in particular. Already in the early period of Polyaesthetic Education, the author of this article [24] identified the huge therapeutic potential inherent in sound scene improvisation, hence its recommendation to initiate self-actualisation in individuals with ODD.

\subsubsection{Self-adjustment}

Apart from diversely discussed pharmacological interventions, e.g. atypical neuroleptics or risperidone, standard therapies to treat ODD include a broad spectrum of psychological approaches such as cognitive-behavioural therapy, parent-child interaction therapy, collaborative problem solving and lifestyle changes. Although self-adjustment as suggested in this articles may look similar, there is still a striking difference: the goal of our approaches is not to readjust one's self in order to better match social expectations, but to identify critical discrepancies between the outer and the inner worlds, and thus to be able to adjust one's behaviour without harming one's personality.

Viable approaches include music-induced altered states of consciousness combined with music-guided imagination techniques, which are designed to help individuals explore pathogenic incompatibilities between personality and sociocultural environments. Increased trance-related creative flow may help to bring about individually adequate 
changes of cognitive and behavioural patterns, and enhance affect- and impulse-oriented self-regulation. Some of these methods resemble sound-related practices of zen-meditation or vocal immersion techniques.

\section{Conclusion}

This model-generating study is part of a huge Chinese project to improve mental health in children and adolescence and to alleviate syndromes through arts-based interventions in educational areas. The relevant spectrum includes, according to Chinese psychiatric epidemiology and mental public health studies, most prevalent conditions in the Chinese younger generation, in addition to ODD namely attention deficit hyperactivity disorder, anxiety disorders, depression, stress and burnout syndromes, eating disorders, and mental disturbances caused by measures to control the COVID-19 pandemic such as social isolation.

Next steps include qualitative exploratory studies, studies to generate standardised models and quantitative multicentre studies, as well as feasibility studies which shall help to provide these educational-therapeutic facilities throughout the People's Republic of China. Moreover, cross-cultural studies shall help to adapt them in different cultures and contribute to intermodal culturally sensitive creative and aesthetic therapies.

\section{Compliance with ethical standards}

\section{Acknowledgments}

This research received no specific grant from any funding agency in the public, commercial or not-for-profit sectors.

\section{Disclosure of conflict of interest}

The author declares no conflict of interest.

\section{Statement of ethical approval}

No approval is needed for this type of research.

\section{References}

[1] https://news.cctv.com/2021/07/23/ARTIMFv0GMfgZPVBnLwuLnNS210723.shtml.

[2] Shen YM, Chan BSM, Liu JB, Zhou YY, Cui XL, He YQ, Fang YM, Xiang YT, Luo XR. The prevalence of psychiatric disorders among students aged 6 - 16 years old in central Hunan, China. BMC psychiatry. 2018; 18(1): 243.

[3] Canino G, Polanczyk G, Bauermeister JJ, Rohde LA, Frick PJ. Does the prevalence of CD and ODD vary across cultures? Social psychiatry and psychiatric epidemiology. 2010; 45(7): 695-704.

[4] Lin X, Li L, Heath MA, Chi P, Xu S, Fang X. Multiple levels of family factors and oppositional defiant disorder symptoms among Chinese children. Family process. 2018; 57(1): 195-210.

[5] Quay HC. Dimensions of personality in delinquent boys as inferred from the factor analysis of case history data. Child development. 1964; 35: 479-84.

[6] Jenkins RL, Boyer A. Types of delinquent behavior and background factors. International journal of social psychiatry. 1967-1968; 14(1): 65-76.

[7] Pardini DA, Frick PJ, Moffitt TE. Building an evidence base for DSM-5 conceptualizations of oppositional defiant disorder and conduct disorder: introduction to the special section. Journal of abnormal psychology. 2010;119(4): 683-688.

[8] Riley M, Ahmed S, Locke A. Common questions about oppositional defiant disorder. American family physician, 2016; 93(7): 586-591.

[9] Salvatore JE, Dick DM. Genetic influences on conduct disorder. Neuroscience and biobehavioral reviews. 2018; 91: 91-101.

[10] Mikolajewski AJ, Taylor J, Iacono WG. Oppositional defiant disorder dimensions: genetic influences and risk for later psychopathology. Journal of child psychology and psychiatry. 2017; 58(6): 702-710. 
[11] Lowet DS, Kolan A, Vaida F, Hesselink JR, Levin HS, Ewing-Cobbs L, Schachar RJ, Chapman SB, Bigler ED, Wilde EA, Saunders AE, Yang TT, Tymofiyeva O, Arif H, Max JE. Novel oppositional defiant disorder 6 months after traumatic brain injury in children and adolescents. Journal of neuropsychiatry and clinical neurosciences. 2021 [Epub ahead of print].

[12] Curtis DF, Elkins SR, Areizaga M, Miller S, Brestan- Knight E, Thornberry T. Oppositional defiant disorder. In: Kapalka GM, ed. Disruptive disorders and behaviors: a concise guide to psychological, pharmacological and integrative treatments. New York: Routledge; 2015; 99-119.

[13] Mastnak W. Coherence size and confidence range: two new parameters in psycho-cardiology. Frontiers of cardiology \& cardiovascular medicine. 2021; 1(1): 1-17.

[14] Mastnak W. Systemic Meta-Synthesis. ResearchGate.

[15] Evans SC, Pederson CA, Fite PJ, Blossom JB, Cooley JL. Teacher-reported irritable and defiant dimensions of oppositional defiant disorder: social, behavioral, and academic correlates. School mental health. 2016; 8: 292304.

[16] Gadow KD, Drabick DA. Symptoms of autism and schizophrenia spectrum disorders in clinically referred youth with oppositional defiant disorder. Research in developmental disabilities. 2012; 33(4): 1157-1168.

[17] Ghosh A, Ray A, Basu A. Oppositional defiant disorder: current insight. Psychology research and behavior management. 2017; 10: 353-367.

[18] Cohen JL, Johnson JL, Orr PP. Video and filmmaking as psychotherapy: research and practice. Routledge: 2015.

[19] Powell M. Cinematherapy as a clinical intervention: theoretical rationale and empirical credibility. University of Arkansas, Fayetteville: Theses and dissertations. 2008.

[20] Mastnak W. Maurice Ravels „L'Enfant et les sortilèges“. Moraloper, psychoanalytisches Lehrstück oder magischsinnliches Phänomen? In: Roscher W; Institut für Integrative Musikpädagogik und Polyästhetischer Erziehung, eds. Wagnis der Bildung. Klänge - Texte - Bilder - Szenen. 50 Jahre nach Kriegsende und Holocaust (Jahrbuch Polyaisthesis Bd. 5). München/Salzburg: Katzbichler; 1996; 126-132.

[21] Palermo MT, Di Luigi M, Dal Forno G, Dominici C, Vicomandi D, Sambucioni A, Proietti L, Pasqualetti P. Externalizing and oppositional behaviors and karate-do: the way of crime prevention. A pilot study. International journal of offender therapy and comparative criminology. 2006; 50(6): 654-660.

[22] Mastnak W. Sport identity - Key incentives to adhere to sports-based prevention and rehabilitation. Deutsche Zeitschrift für Sportmedizin (German journal of sports medicine). 2018; 69(4): 38-44.

[23] Allesch C, Schwarzbauer M. (2009). Polyaesthetic Education: A model for integrating cultural experiences. In: Erzen JN ed. Congress book II. Selected papers XVII th International Congress of Aesthetics. Ankara: Sanart; 2009; 19-27.

[24] Mastnak W. Psychodrama und Klangszenenimprovisation. In: Roscher W, ed. Sinn und Widerspruch musikalischer Bildung. Beiträge zu „poiesis“ und „aisthesis“ heute. München/Salzburg: Katzbichler. 1993; 105130. 